\title{
Use of magnetocrystalline anisotropy in spin-dependent tunneling
}

\author{
R. A. Lukaszew, ${ }^{\text {a) }}$ Y. Sheng, C. Uher, and R. Clarke \\ Randall Laboratory of Physics, University of Michigan, Ann Arbor, Michigan 48109-1120
}

(Received 1 June 1999; accepted for publication 27 July 1999)

\begin{abstract}
Epitaxial growth techniques are used to impose in-plane magnetocrystalline anisotropy on a spin-polarized tunneling configuration. A $\mathrm{Cu}(100)$ buffer layer grown on a $\mathrm{Si}(100)$ substrate stabilizes epitaxial face-centered-cubic cobalt as one of the ferromagnetic electrodes. The negative magnetocrystalline constant of this metastable phase favors easy axes along Co $\langle 110\rangle$ and, due to the single crystal nature of this layer, the coercivity is more than an order of magnitude larger than in the polycrystalline layers which form the second electrode. Our approach provides a way to access the high degree of spin polarization characteristic of the $3 d$ transition metals. (C) 1999 American Institute of Physics. [S0003-6951(99)00139-4]
\end{abstract}

It has been known for some time that magnetic thin films can exhibit abrupt switching in their magnetization state as a function of magnetic field. ${ }^{1}$ This behavior is accompanied by the existence of symmetry-related preferred directions (or "easy" axes) for the magnetization vector determined by the magnetic anisotropy. There are important consequences for recently developed magnetic nanostructures whose relevant properties are controlled entirely by their magnetization state.

An interesting example is the magnetic tunnel junction (MTJ) consisting of a pair of weakly coupled ferromagnetic (FM) electrodes separated by a thin insulating barrier. ${ }^{2-4}$ In this case, an asymmetry in the density of states of the majority and minority bands in the FM electrodes leads to a spindependent tunneling probability such that parallel (antiparallel) magnetization configurations of the two electrodes leads to high (low) conduction through the barrier. ${ }^{5-7}$ Because of the collective nature of this magnetoresistance effect, there is reason to believe that magnetocrystalline anisotropy would be an important parameter in the behavior of spin-dependent tunneling systems. To date, this aspect of MTJ structures has largely been ignored as most of the work has been carried out on polycrystalline samples.

In this work we report the use of epitaxial growth techniques to impose a magnetocrystalline anisotropy on a spinpolarized tunneling device. Our approach establishes a welldefined crystal structure for the MTJ, providing a convenient way to access the high degree of spin-polarization characteristic of $3 d$ transition metals.

The samples were prepared by a combination of molecular-beam epitaxy (MBE) (metallic ferromagnetic layers) and electron cyclotron resonance (ECR)-assisted plasma deposition (insulating layers). A Cu (3000 ̊) single crystalline seed layer was first grown on a $\mathrm{Si}(001)$ substrate at a rate of $\sim 0.3 \AA / s$ in several stages with intermediate mild annealings to achieve a smooth surface. ${ }^{8}$ This $\mathrm{Cu}$ single crystal film grew epitaxially in the (100) orientation with an inplane rotation angle of $45^{\circ}$ with respect to the $\mathrm{Si}$ lattice, serving as a template to stabilize the subsequent growth of single crystal, face-centered-cubic (fcc) Co. Single crystal

a)Electronic mail: lukaszew@umich.edu
Co(100) films exhibit a strong negative fourfold in-plane anisotropy that favors $\langle 110\rangle$ as the easy axis of magnetization. ${ }^{9}$

Reflection high-energy electron diffraction (RHEED) oscillations were used to monitor the layer-by-layer growth of the fcc Co electrode to a thickness of approximately $60 \AA$. The insulating barrier layer consisted of highly oriented hexagonal-close-packed (hcp) boron nitride $(\mathrm{BN})$ grown on fcc Co to an average thickness of $20-30 \AA$. BN is a relatively new material for this application. It has a wide band gap $(\sim 5 \mathrm{eV})$ comparable to that of aluminum oxide $(9 \mathrm{eV})$, which is the insulating material used in most magnetic tunneling junctions. ${ }^{5-7} \mathrm{BN}$ has an important advantage over $\mathrm{Al}_{2} \mathrm{O}_{3}$, namely that the possibility of partially oxidizing the magnetic layers during the fabrication process is greatly reduced. A second magnetic layer $(\sim 60 \AA$ of $\mathrm{Co}$ or $\mathrm{Ni})$ was then deposited in the same manner as the first layer, but in the absence of a single crystal $\mathrm{Cu}$ template this layer grew polycrystalline. The samples were capped with $50 \AA$ A of copper to protect them from atmospheric degradation.

In addition to in situ RHEED, the structure of the sample was characterized via ex situ cross-sectional transmission electron microscopy (TEM). Figure 1 shows a TEM image illustrating that the insulating layer is continuous and relatively flat, with no observable pinholes. The inset in Fig. 1 shows a high resolution TEM (HRTEM) image of a test sample with this kind of structure confirming the crystallographic structure of the various layers as previously determined by RHEED. Fourier transform analysis of the HRTEM images indicated fcc structure for the initial $\mathrm{Cu}$ and Co layers, textured growth (with the basal planes normal to the substrate) for the BN layer, and polycrystalline structure for the second layer (with hcp structure in the case of Co, and fcc structure for $\mathrm{Ni}$ ).

The process of magnetization reversal was studied using the magneto-optic Kerr effect (MOKE) with the magnetic field $B_{\text {ext }}$ applied along the easy $\langle 110\rangle$ in-plane axis of the fcc layer. Figure 2(a) displays a typical magnetic hysteresis loop (MHL) measured at room temperature. The magnetization curve exhibits two intermediate plateaux corresponding to approximately zero net magnetization where the magnetization vectors of the two electrodes are in the antiparallel configuration. The weak coupling between the magnetic layers 


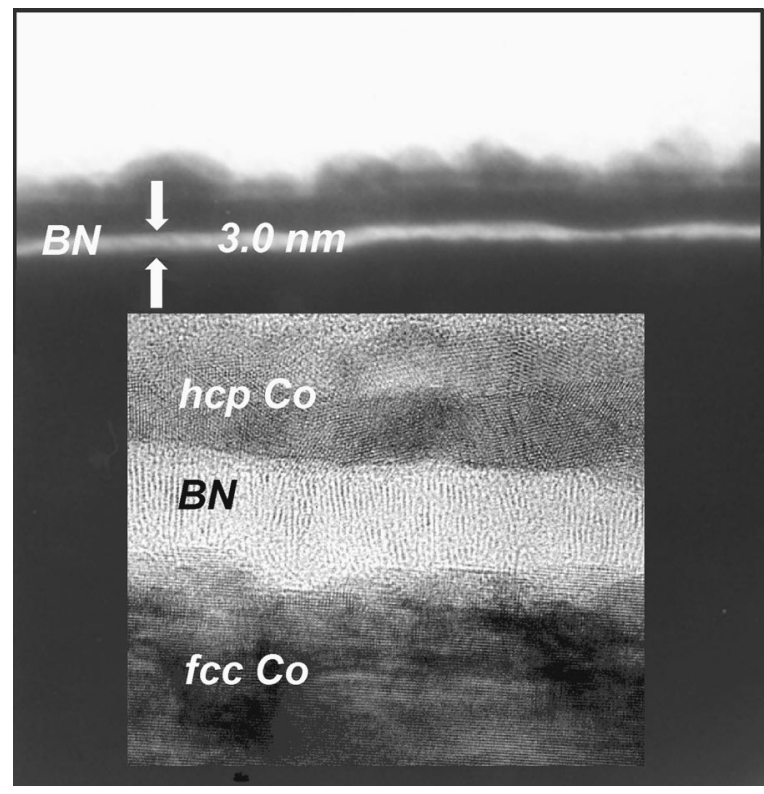

FIG. 1. TEM micrograph showing cross-sectional view of the multilayered structure. Note that the insulating layer is smooth and pin-hole free, with $\sim 50 \%$ thickness variation. Inset: HRTEM showing the crystallographic structure of a test sample.

permits the identification of the contributions to the MHL from each individual layer. Thus, we observe a sharp flip of the magnetization at higher field (150 Oe), with square edges in the hysteresis loop, and a more gradual transition at a lower field $(\sim 10 \mathrm{Oe})$, with rounded edges. The sharp transition corresponds to the typical switching along the easy axis for a single crystal, in this case fcc $\mathrm{Co}$, and the smooth magnetization reversal corresponds to the polycrystalline layer (hcp Co or fcc Ni).

We have proposed a simple model to predict the coercivity $\left(H_{c}\right)$ along the easy axis for an fcc film. In this model the magnetization is allowed to jump between available energy minima in the energy landscape for $H=H_{c}$ when the gain in energy is greater than the energy necessary for domain-wall nucleation. Thus, our predicted $H_{c}$ is $150 \mathrm{Oe}$ for the fcc Co electrode (assuming cross-tie domain wall nucleation, $2 K_{1} / M_{s}$ from Naik et al. ${ }^{10}$ and bulk value for the saturation magnetization) in agreement with the observed value. The details of this model can be found elsewhere. ${ }^{11}$ For polycrystalline materials the magnetization curve is the result of an average over different oriented crystallites. In addition, the crystallite boundaries act as obstacles to domain motion, or for domain rotation, and the hysteresis loop should have a rounded appearance. The coercive field of the polycrystalline layer should be similar to that for bulk materials $\left(\sim 2\right.$ Oe for $\mathrm{Ni}, \sim 9$ Oe for $\mathrm{Co}$, at ambient temperature ${ }^{12}$ ) and that is indeed what we observe. The hysteresis loops for the individual layers were also measured [Figs. 2(b) and 2 (c)] and further confirmed our identification of the components in the composite loop.

An added advantage of this type of sample is the presence of well defined easy and hard axes arising from the crystallographic symmetry given by the in-plane fourfold symmetry of the single crystal fcc layer. Further magnetic characterization at temperatures ranging from 5 to $350 \mathrm{~K}$ was performed using a Quantum Design superconducting quan-
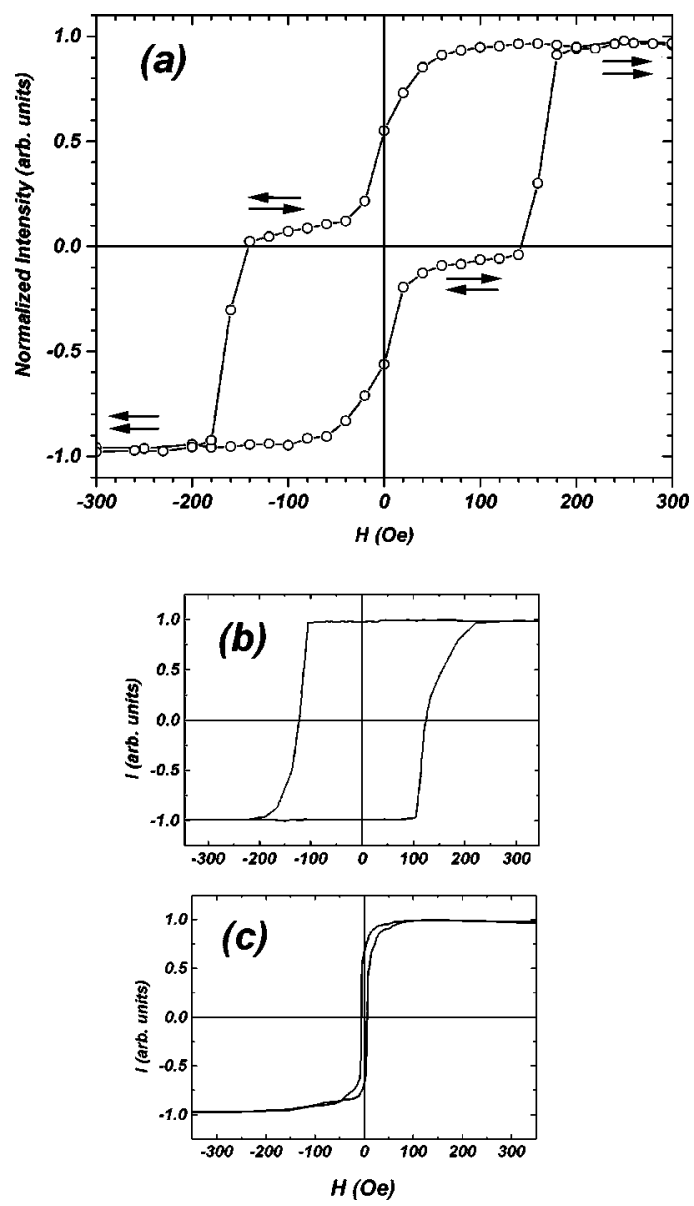

FIG. 2. MOKE magnetic hysteresis loops at room temperature (a) for a spin-tunneling junction with $B_{\text {ext }}$ applied along the easy $\langle 110\rangle$ in-plane axis of the single crystal fcc layer; (b) contribution from the fcc Co layer, with $B_{\text {ext }}$ along $\langle 110\rangle$; (c) from the polycrystalline Co layer.

tum interference device (SQUID) magnetometer. ${ }^{13}$ These measurements confirmed the MOKE data at room temperature.

The samples were patterned lithographically to obtain junctions of square shape with side lengths ranging from 20 to $200 \mu \mathrm{m}$ to minimize the contributions to the resistance that are not magnetic field dependent. Transport properties were measured applying a dc voltage at room temperature, using a two-probe technique. The parabolic behavior of the conductance vs $V$ curve obtained was considered a signature of tunneling. From a fit of the Simmons model ${ }^{14}$ to the current-voltage $(I-V)$ curve near zero, we found that the effective thickness and height of the BN barrier were $1.4 \mathrm{~nm}$ and $1.25 \mathrm{eV}$ respectively.

The barrier height value for $\mathrm{BN}$ appears to be somewhat lower than that reported for aluminum oxide junctions ${ }^{15}$ implying that the absolute resistance values of our MTJs should also be lower than those previously reported for $\mathrm{Al}_{2} \mathrm{O}_{3}$ junctions. Our measurements support this. Figure 3 shows the magnetoresistance of one of these samples (Co fcc bottom electrode, $\mathrm{Ni}$ polycrystal top electrode, junction size: $50 \mu \mathrm{m} \times 50 \mu \mathrm{m})$. A sharp $25 \%$ change in the magnetoresistance was observed at room temperature, at the same fields where the switching of the magnetization was observed in the hysteresis loop. The magnetotunneling effect thought to be operative can be explained using a model proposed by 


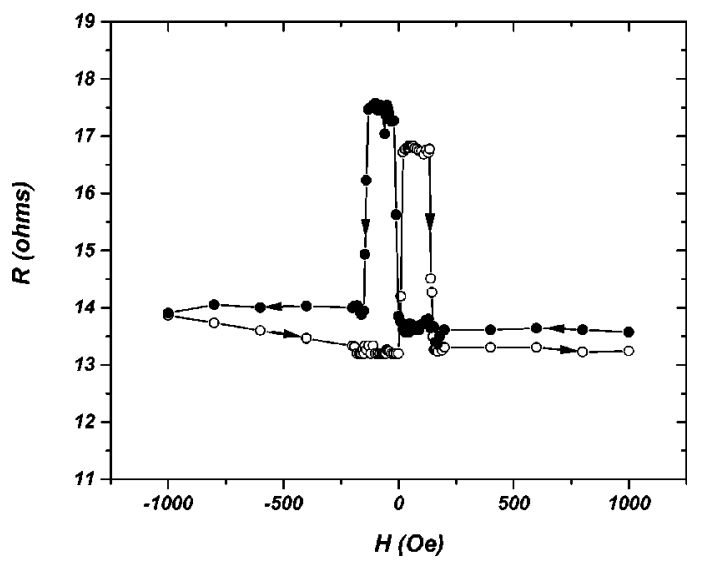

FIG. 3. Room temperature MR with $B_{\text {ext }}$ applied along the easy $\langle 110\rangle$ inplane axis of the fcc single crystal layer. (Sample composition: Co fcc/ $\mathrm{BN} / \mathrm{Ni}$ polycrystal. Junction size: $50 \mu \mathrm{m} \times 50 \mu \mathrm{m}) . \Delta R / R \simeq 25 \%$.

Tedrow and Meservey. ${ }^{3}$ This model considers the asymmetry in the density of states of the majority and minority energy bands in a ferromagnet and assumes that the spin is conserved during the tunneling process. The magnetoresistance ratio is given by

$$
\Delta R / R_{p}=R_{\text {ap }} / R_{p}-1=2 P_{1} P_{2} /\left(1-P_{1} P_{2}\right),
$$

where $R_{\text {ap }}$ is the resistance of the junction with antiparallel alignment of the magnetizations in the two FM electrodes, and $R_{p}$ is the resistance with the magnetizations aligned parallel. $P_{1}$ and $P_{2}$ are the spin polarization coefficients of electrodes 1 and 2, respectively. Other theories have been proposed to extend the previous model and make it more rigorous. Slonczewski predicts that the barrier height and the character of the FM-I interface should also have an influence on the MR. ${ }^{4}$

From application of Eq. (1) we expect an optimum magnetoresistance ratio of $28 \%$ for Co-Co samples and $17.5 \%$ for Co-Ni samples $\left(P_{\mathrm{Co}}=0.35 \pm 0.03, P_{\mathrm{Ni}}=0.23 \pm 0.03\right.$ from Ref. 3). Surprisingly the observed change $(25 \%)$ is somewhat larger than the maximum value expected within the experimental uncertainties associated with $P_{\mathrm{Co}}$ and $P_{\mathrm{Ni}}$ mentioned in Ref. 3, for samples using aluminum oxide as tunneling barriers. On the other hand Upadhyay et al. ${ }^{16}$ have recently used Andreev reflection to measure the spin polarization of the direct current in FM-superconductor thin films prepared with abrupt oxide-free interfaces. They report polarization values of $P_{\mathrm{Co}}=0.37$ and $P_{\mathrm{Ni}}=0.32$, and attribute the difference between these values and those in Ref. 3 to the fact that their measurements relate to the polarization in the bulk current rather than being influenced by interface effects, as could be the case in the tunneling measurements. Using values of Ref. 16 in Eq. (1) we get a magnetoresistance ratio of $27 \%$, in better agreement with our experimental value. Thus our results seem to indicate that the spin-polarized tunneling in our samples is also determined by a high degree of polarization in the ferromagnetic layers. The absence of oxidation processes that may also oxidize the ferromagnetic surface in contact with the insulating layer thus preserves the magnetic character of the interface.

In summary, we have used epitaxial growth techniques to impose in-plane magnetocrystalline anisotropy on structures intended for spin-tunneling applications. The samples consisted of two MBE-deposited magnetic layers with different magnetic properties due to their different crystallographic structure, providing well-defined and separated values of $H_{c}$. One layer was grown as single crystalline fcc Co while the other magnetic layer was polycrystalline (hcp Co or fcc $\mathrm{Ni}$ ). This approach has the added advantage of the presence of easy and hard axes arising from the in-plane fourfold anisotropy of the single crystal fcc Co layer. The use of BN as a barrier layer avoids potential oxidation problems at the FM-I interface, implying spin-polarization values that agree with those measured recently by Andreev reflection techniques. Moreover the apparently smaller barrier height of the $\mathrm{BN}$ insulating layer is favorable for lowering the absolute resistance of magnetic tunnel junctions.

Work supported in part by ONR Grant No. N00014-92$\mathrm{J}-1335$.

${ }^{1}$ K. B. Hathaway and G. Prinz, Phys. Rev. Lett. 47, 1761 (1981).

${ }^{2}$ M. Julliere, Phys. Lett. A 54, 225 (1975).

${ }^{3}$ R. Meservey and P. M. Tedrow, Phys. Rep. 238, 173 (1994).

${ }^{4}$ J. C. Slonczewski, Phys. Rev. B 39, 6995 (1989).

${ }^{5}$ J. S. Moodera, L. R. Kinder, T. M. Wong, and R. Meservey, Phys. Rev. Lett. 74, 3273 (1995); J. S. Moodera, L. R. Kinder, J. Nowak, P. LeClair, and R. Meservey, Appl. Phys. Lett. 69, 708 (1996).

${ }^{6}$ W. J. Gallagher, S. S. P. Parkin, Yu Lu, X. P. Bian, A. Marley, K. P. Roche, R. A. Altman, S. A. Rishton, C. Jahnes, T. M. Shaw, and G. Xiao, J. Appl. Phys. 81, 3741 (1997).

${ }^{7}$ J. F. Bobo, F. B. Mancoff, K. Bessho, M. Sharma, K. Sin, D. Guarisco, S. X. Wang, and B. M. Clemens, J. Appl. Phys. 83, 6685 (1998).

${ }^{8}$ R. A. Lukaszew, Y. Sheng, C. Uher, and R. Clarke (unpublished).

${ }^{9}$ B. Heinrich, J. F. Cochran, H. Kowaleswski, J. Kirschner, Z. Celinski, A. S. Arrott, and K. Myrtle, Phys. Rev. B 44, 9348 (1991).

${ }^{10}$ R. Naik, C. Kota, J. S. Payson, and G. L. Dunifer, Phys. Rev. B 48, 1008 (1993).

${ }^{11}$ R. A. Lukaszew, E. Smith, Y. Sheng, R. Naik, C. Uher, and R. Clarke (unpublished).

${ }^{12}$ Ferromagnetism, edited by R. B. Bozorth (Van Nostrand, Princeton, New Jersey, 1968), pp. 267-273.

${ }^{13}$ L. Pust, L. Wenger, R. A. Lukaszew, Y. Sheng, D. Litvinov, Y. Wang, C. Uher, and R. Clarke, J. Appl. Phys. 85, 5765 (1999).

${ }^{14}$ J. G. Simmons, J. Appl. Phys. 34, 1793 (1963).

${ }^{15}$ J. S. Moodera, E. F. Gallagher, K. Robinson, and J. Nowak, Appl. Phys. Lett. 70, 3050 (1997).

${ }^{16}$ S. K. Upadhyay, A. Palarisami, R. N. Louie, and R. A. Buhrman, Phys. Rev. Lett. 81, 3247 (1998). 